\title{
Cystic fibrosis, elevated gamma-glutamyltransferase, and lung transplant outcome
}

doi:10.1111/j.1432-2277.2012.01563.x

Dear Sirs,

In their study recently published in this journal [1], Wree et al. have investigated the prognostic impact of liver function tests in patients with end-stage cystic fibrosis (CF) undergoing double lung transplant (DLTx). The authors' focus was put on CF-associated liver injury as factor possibly affecting the outcome of surgery even in absence of overt signs of liver cirrhosis, such as portal hypertension. In a cohort of 31 cases with median followup of 1000 days, preoperatively elevated gamma-glutamyltransferase (GGT) was identified to be associated with higher mortality following DLTx. Postoperatively elevated GGT was also associated with higher mortality, accompanied in this case by elevated bilirubin. Thus, the importance of close monitoring of liver function in $\mathrm{CF}$ candidates to DLTx is underscored [1].

We would like to call the attention on a possible alternative explanation for elevated serum GGT levels in CF patients. Despite the well-known and established role of GGT as a marker of liver and biliary disease, the significance of this enzyme activity in fact has recently expanded to include several additional aspects, e.g., in tumor biology [2] and atherosclerosis [3]. As far as CF is concerned, GGT is known to increase several fold in the lung lining fluid of CF patients [4]. In CF sputum specimens, we have recently identified activated neutrophilic granulocytes as the source of GGT increase; neutrophils in fact contain GGT in their specific granules and can release it upon activation [5]. Identification of the neutrophilic origin of increased GGT was achieved by means of chromatographic techniques allowing to separate and compare distinct GGT-containing protein fractions deriving from different cellular sources [6].

It has been suggested that CF neutrophils may have an increased propensity to release their granule proteins, because of increased secretion of cytokines and/or still unknown priming mechanisms [7]. It would be of interest to verify whether the increased serum GGT observed by Wree et al. might also derive from hyperreactivity of peripheral blood neutrophils in selected patients. Rather than referring to underlying conditions of liver dysfunction, the less favorable outcome of DLTx in these subjects could point then to a "hyperinflammatory" status [8], which could prompt the devisement of more specific pre- and/or postoperative therapeutic strategies.

\section{Alessandro Corti, ${ }^{1}$ Maria Franzini, ${ }^{2}$ Silvana Cianchetti, ${ }^{3}$ Pierluigi Paggiaro ${ }^{3}$ and Alfonso Pompella ${ }^{1}$ \\ 1 Dipartim. di Patologia Sperimentale, Scuola Medica dell'Università di Pisa, Pisa, Italy \\ 2 Scuola Superiore S. Anna di Studi Universitari e di Perfezionamento, Pisa, Italy \\ 3 Dipartim. Cardio-toracico e Vascolare, Scuola Medica dell'Università di Pisa, Pisa, Italy e-mail: alfonso.pompella@med.unipi.it}

\section{Conflict of interest}

The authors declare no existing conflict of interest.

\section{Funding}

The financial support by the Fondazione Fibrosi Cistica (Verona, Italy) is gratefully acknowledged.

\section{References}

1. Wree A, Bechmann LP, Kumarasamy N, et al. Elevated gamma-glutamyltransferase is associated with mortality in lung transplantation for cystic fibrosis. Transpl Int 2012; 25: 78 .

2. Pompella A, Corti A, Paolicchi A, Giommarelli C, Zunino F. Roles of gamma-glutamyltransferase in cell redox regulation, drug resistance and cancer progression. Curr Opin Pharmacol 2007; 7: 360.

3. Franzini M, Corti A, Martinelli B, et al. Gamma-glutamyltransferase activity in human atherosclerotic plaques biochemical similarities with the circulating enzyme. Atherosclerosis 2009; 202: 119.

4. Galli F, Battistoni A, Gambari R, et al. Oxidative stress and antioxidant therapy in cystic fibrosis. Biochim Biophys Acta 2011; 1822: 690.

5. Corti A, Franzini M, Cianchetti S, et al. Contribution by polymorphonucleate granulocytes to elevated 
gamma-glutamyltransferase in cystic fibrosis sputum. PLoS One 2012; 7: e34772.

6. Franzini M, Bramanti E, Ottaviano V, et al. A high performance gel-filtration chromatography method for gammaglutamyltransferase fraction analysis. Anal Biochem 2008; 374: 1 .
7. Koller DY, Urbanek R, Götz M. Increased degranulation of eosinophil and neutrophil granulocytes in cystic fibrosis. Am J Respir Crit Care Med 1995; 152: 629.

8. Machen TE. Innate immune response in CF airway epithelia: hyperinflammatory? Am J Physiol Cell Physiol 2006; 291: C218. 